\title{
A numerical study on the hydrodynamic impact of device slenderness and array size in wave energy farms in realistic wave climates
}

\author{
Markel Penalba ${ }^{\mathrm{a}, *}$, Imanol Touzón ${ }^{\mathrm{b}, \mathrm{c}}$, Joseba Lopez-Mendia ${ }^{\mathrm{b}}$, Vincenzo Nava ${ }^{\mathrm{b}}$ \\ ${ }^{a}$ Center for Ocean Energy Research, Maynooth University \\ Maynooth, Co. Kildare, Ireland \\ ${ }^{b}$ Tecnalia Research and Innovation \\ Parque Tecnologico de Bizkaia, Edificio 700 \\ 48160, Derio, Bizkaia, Spain \\ ${ }^{c}$ Department of Mechanical Engineering \\ University of the Basque Country (EHU/UPV)
}

\begin{abstract}
The future of wave energy converters lies in the design and realization of farms comprising of several devices, given the level of actual power flow for the individual devices and because of several operational issues. Therefore, not only the hydrodynamics of individual and isolated devices should be analysed, but interactions among devices within an array must also be carefully evaluated. In this paper, the authors attempt to parameterize the behaviour of small-, mediumand large-arrays of wave energy converters, in a particular staggered configuration, at four different locations characterized by realistic wave climates. The arrays studied in the present paper consist of heaving cylinders of different slenderness ratios. It turns out that for arrays of very short inter-device distances, regardless of the cylinder and array size, interactions are strong and lead to not negligible effects of destructive interference (total power reduction compared to the sum of isolated devices). Under these conditions, the bigger the array, the stronger the interactions and the higher the loss of power. However, a range of inter-device distances, referred to as intermediate region, where the power absorption is consistent and the interaction effect appears to be positive, has
\end{abstract}

\footnotetext{
* Corresponding author

Email address: mpenalba@eeng.nuim.ie ( Markel Penalba)
} 
been found. This intermediate region is easily detectable for small arrays, but loses its ideal characteristics with the increase of the size of the array.

Keywords: Wave energy converter, wave energy array, farm layout, wave interaction, inter-device distance

\section{Introduction}

Since the awareness of the exhaustion of traditional energy resources and the irreversible environmental impacts from fossil fuel combustion has increased, renewable and carbon-emission-free resources have been investigated intensively, 5 with some resources already participating in the energy mix.

In this respect, wave energy may become an important renewable resource, as shown in [1], if the existing technologies develop sufficiently. Many different concepts of wave energy converters (WECs), based on diverse working principles (e.g. heave point absorbers, oscillating wave sure converters or pitch attenuators) have been developed during the last decades, mainly focusing on individual devices. Heave point absorbers are floating bodies, whose horizontal extent is much smaller than a wavelength [2]. They absorb wave energy through their movement at the free-surface and the conversion into electrical power can be achieved through different power take-off (PTO) systems. In detail, the hydrodynamic analysis of single point absorbers is usually carried out using the well known boundary element method (BEM) theory, because of the wide availability of several commercial or open-source codes, such as WAMIT [3], AQWA [4] or NEMOH [5], the relative ease of use and its appealing computational costs.

However, due to the actual power flow and high costs derived from construction, installation and maintenance of WECs, it seems that the only viable option is to incorporate more devices into 'wave farms'. It is therefore important to understand not only the behaviour of an isolated device, but also the interactions among the devices in a farm.

Hydrodynamic interactions in WEC arrays have been studied since the 1970's, when 6] introduced the concept of point absorber for array interactions 
and [7] suggested an expression for the power absorbed by a WEC array. Different semi-analytical methods have been suggested to efficiently compute the hydrodynamic interactions within WEC arrays, such as the plain-wave method or the multiple scattering method introduced by [8] and [9]. Another alternative 30 is the direct matrix method presented by [10]. All the aforementioned methods are based on the linear theory and provide exact solutions under certain assumptions.

More recent works analyse such hydrodynamic interactions both numerically and experimentally: [11] and [12] analyse numerically the hydrodynamic interactions as a function of different inter-device distances for different array configurations, including very large separating distances of over $2000 \mathrm{~m}$, while [13] investigates experimentally the interactions in large arrays.Some effort has also been dedicated in methods for array layout optimisation, for example [14] or [15], which consider wave directionality and array layout, or a more recent 40 study [16], based on the hydrodynamic model recently presented by [17], considering six different parameters to optimise the layout. [18] presents an overview of the different methods to analyse WEC arrays and a whole section is given to WEC array modelling techniques in [19].

So far, most of the work for the analysis of the interaction among devices in 45 a wave energy array has been carried out under regular wave conditions. Nevertheless, a more detailed approach is needed in order to accurately study the hydrodynamic interactions. For this reason, there is a gradual move in the literature towards studying such interactions in spectral seas: 20] studies cylindrical heaving bodies of different geometries in two different array configurations at the including sub-optimal control in WEC arrays at the European Marine Energy Centre (EMEC), comparing results to those obtained under regular waves, and 22] studies several different configurations using the scatter-diagram information at Yeu Island in France.

55

The size of arrays may also be important, so large arrays have been studied in some works in the literature, such as, 23] which studies 18 SEAREV devices 
in the array, 24] which studies 25 cylinders and 25 surging barges or [25] which studies 32 AWS devices.

[26] presents different factors that influence the behaviour of wave energy devices in an array, including the array configuration, the inter-device distance, the number of devices in the array and the incident direction of the wave. However, arrays of only 2-4 WECs are investigated, which may lead to incomplete and/or misleading conclusions. In addition, the geometry of the devices, particularly the slenderness ratio (radius/draft) in axisymmetric devices as shown in [20, and characteristics of the incoming waves may also influence the behaviour of the WECs in the array.

In this paper, the influence of the slenderness and the number of devices in a wave farm on the hydrodynamic performances is evaluated numerically in realistic wave climates, as function of inter-device distance. Scatter diagrams of four different locations, representative of various resource distributions, have been used in the analysis.

Section 2 introduces the hydrodynamic model used in the simulations, Section 3 describes different device geometries, the array layout configuration, array sizes and the locations, while Section 4 shows the results for each case. Finally, conclusions are drawn in Section 5.

\section{Hydrodynamic model}

The interaction between wave absorbers and fluid has very often been modelled by means of the linear diffraction theory, under the assumption of inviscid fluid and incompressible irrotational flow. In this study, linear theory has been considered, assuming wave and body motion amplitudes to be small with respect to the wavelength, and allowing the formulation of the solution of the boundary conditions and Bernoullis equation in terms of velocity potential and free surface displacement. The influence of nonlinear hydrostatics and Froude-Krylov forces for assessing the absorption of wave energy is still under investigation in order to define appropriate ranges of validity even if evidence of their influ- 
ence on dynamics of bodies is well-known [27. Nevertheless, the same authors suggest that the linearization of the free surface condition is consistent with the basic definition of point absorber (main dimension much smaller than the wavelength), and of course the effects are increasing with the wave amplitudes.

90 Similarly, nonlinear radiation effects seem to be not so relevant. For all these reasons (small size of the device, small amplitudes, minor effects of nonlinear radiation), the linear theory seems to be a good choice for identifying the main characteristics of the interactions among devices, without no major impact on accuracy.

According to 28, indeed, when the bodies are large enough, the flow remains attached to the surface, and therefore, the resulting force on the body can be performed by integration of the pressures. In such cases, Froude-Krylov forces and diffraction and radiation forces can be used for the estimation of forces. When not applicable, other models for the fluid structure interactions should be used, in order to include viscous effects (for example, Morison equation, including viscous drag force, as an inertial term) or proceed to solve full Navier Stokes equations by means of Computational Fluid Dynamics (CFD), which will make the problem very cumbersome from the computational point of view.

In particular, the diffraction model can be applied either when the dimensionless Keulegan-Carpenter (KC) number is lower than a threshold value, with this threshold generally set to the value of 6 , or -following an entirely equivalent interpretation- when the diffraction parameter $\pi \frac{D}{L}$ is greater than 0.5 , where $D$ is the significant dimension for the body (i.e. the diameter for a vertical cylinder) and $L$ is the wavelength. Essentially, following [28, from mild to moderate sea states the linear diffraction model can be applied. Even in extreme sea states, viscous drag forces are negligible when the ratio $\frac{H}{D}$ is lower than 2. Therefore, for the case studies in the present paper, the drag effect is almost negligible, and only the inertial term could be taken into account for the estimation of forces, even when the Morison equation should be used.

All the above considerations yield to the conclusion that diffraction forces cover all the major effects on forces, given the occurrence matrices and scatter 
diagrams in Figure 4

Hydrodynamic coefficients are in this case obtained by using the commercial code AQWA [4. Mesh density for the simulations has been decided after a mesh convergence study for an isolated device, where the best compromise between accuracy and computational costs was found to occur using a mesh of 2016 nodes and 504 panels. The same number of nodes and panels is used for all simulations. In such simulations, a range of 50 frequencies between 0.03 and $2 \mathrm{rad} / \mathrm{s}$ was analysed, which covers the vast majority of the exploitable ocean waves.

In the case studies, waves are modelled as 2D long-crested cylindrical waves, i.e. a unidirectional spectrum without any spreading factor is used in all the simulations, and the incoming waves are always perpendicular to the main direction of array. In undisturbed field, in general, the effects of directional spreading becomes particularly relevant in case of nonlinear waves and shallow water (see [29]). As a matter of fact, the hydrodynamic performance of the array should depend on the incident wave direction and taking into account a directional spreading function may reduce the final power output of the array especially if the devices are aligned with the mean wave direction, as noticed by [20]]. However, given the configuration of the layout of devices studied in this work and the linearity of the wave model adopted, it is reasonable to consider those effects to be of smaller entity and they have been not taken into account within the scope of this work.

\subsection{Single-device}

Once hydrodynamic coefficients are calculated, the equation of motion in frequency-domain is obtained from

$$
-\omega^{2}(M+A(\omega)) \hat{X}+j \omega\left(B(\omega)+B_{P T O}\right) \hat{X}+K_{H} \hat{X}=\hat{F}_{e}(\omega)
$$

where $M$ is the mass, $A(\omega)$ the added-mass, $B(\omega)$ the radiation damping, $\hat{X}$ the position of the body, $\omega$ the wave frequency, $K_{H}$ the hydrodynamic stiffness, 
$B_{P T O}$ the damping value of the PTO force, $\hat{F}_{e}(\omega)$ the excitation force and $j$ the denotes complex number $(j=\sqrt{-1})$. Since a linear system is assumed, if sinusoidal waves are considered, motion is also a sinusoidal function that can be written as a function of time: $\mathrm{x}(\mathrm{t})=\operatorname{Re}\left(\hat{X} e^{j \omega t}\right)$. The same can be applied to the forces. The PTO is modelled as a linear damper and is optimised for each cylinder and sea-state. The absorbed power, $P_{i}(\omega)$, over one wave period is calculated as in Eq. (2) for regular waves and Eq.(4) for irregular waves [30.

$$
P_{i}(\omega)=\frac{1}{2} B_{P T O} \omega^{2}\left|\delta_{i}\right|^{2}
$$

where $\delta_{i}$ is the normalised amplitude of the oscillation in heave, $\delta_{i}=X / H$, being $H$ the wave amplitude. To evaluate the power absorption under irregular waves, the energy (density) spectrum $S(\omega)$ can be defined as follows,

$$
S(\omega)=\frac{1}{2} \frac{A_{s p}^{2}(\omega)}{d \omega}
$$

where $A_{s p}(\omega)$ is the wave amplitude spectrum and the power absorption can be given as

$$
P_{i r}=\int_{0}^{\infty} 2 P_{i}(\omega) S(\omega) d \omega
$$

For irregular waves, it is interesting to analyse the annual mean power, calculated by using the wave data statistics for a given location as a function of the significant wave height $\left(H_{s}\right)$ and peak period $\left(T_{p}\right)$,

$$
\left\langle P_{i}\right\rangle=\sum_{\left(H_{s}, T_{p}\right)} P_{i r} C\left(H_{s}, T_{p}\right)
$$

where $C\left(H_{s}, T_{p}\right)$ is the occurrence of each peak period and significant height in the corresponding location. The same method suggested in the present paper has been used in different studies in the literature to evaluate the power absorption of WEC arrays in irregular waves [11, 20]. However, this methodology is not unique in the literature. For example, 31] suggests a different procedure to evaluate power absorption under irregular waves including wave directionality. 
For the case of $\mathrm{N}$ devices in an array, Eq. (1) can be easily extended by introducing matrix notation

$$
-(\widetilde{M}+\widetilde{A}(\omega)) \omega^{2} \hat{X}+\left(\widetilde{B}(\omega)+\widetilde{B}_{P T O}(\omega)\right) j \omega \hat{X}+\widetilde{K}_{H} \hat{X}=\widetilde{F}_{e}(\omega)
$$

where $\widetilde{A}$ and $\widetilde{B}$ are $\mathrm{NxN}$ symmetric matrices (N being the number of devices in the array, as a single degree of freedom is considered for each device), $\widetilde{M}$ and $\widetilde{K}_{H}$ are diagonal matrices of the same order as $\widetilde{A}$ and $\widetilde{B}$, and $\hat{X}$ and $\widetilde{F}_{e}$ are Nx1 vectors. Matrices $\widetilde{A}$ and $\widetilde{B}$ are symmetric because cross values represent hydrodynamic interaction studied in pairs, where the effect of the device $i$ on the device $j$ and vice versa are the same $\left(a_{i j}=a_{j i}\right)$.

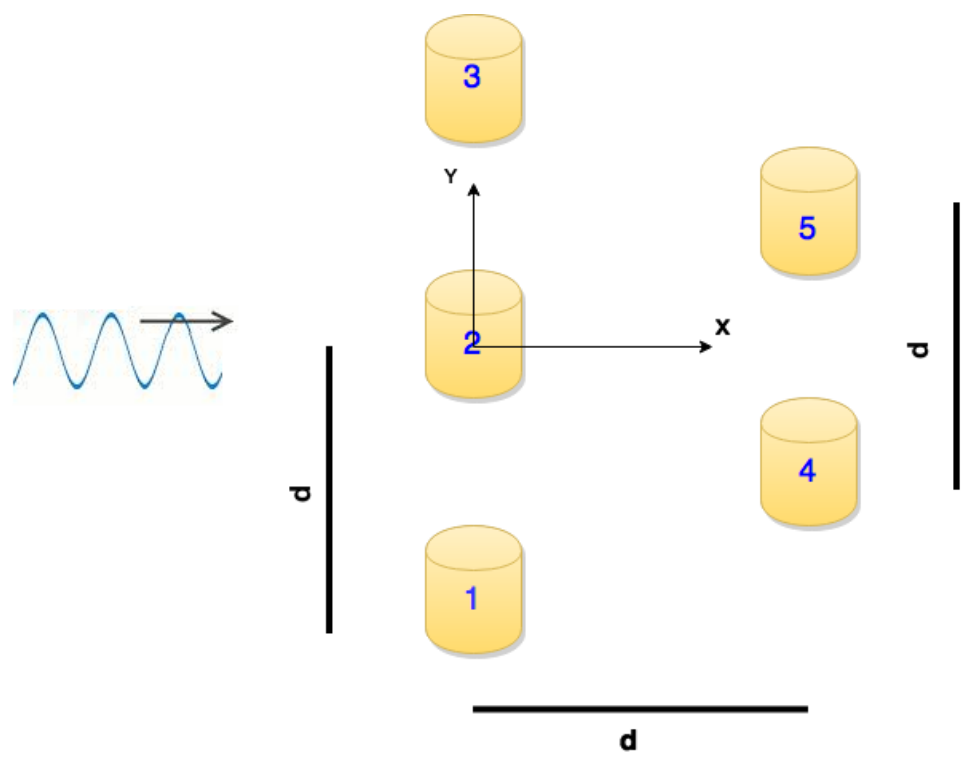

Figure 1: Staggered configuration of the 5 device array

Figure 1 illustrates the case of an array with 5 devices. Hydrodynamic 175 coefficients obtained with the AQWA code for the case of an isolated device and the case with 5 devices are shown in Figure 2. Cylinders of slenderness 
ratio (radius/draft) equal to 1 are used in both isolated and 5-device array shown in Figure 2, where the inter-device spacing in the array is of 5 diameters $(\mathrm{d}=5 \mathrm{D} \sim 125 \mathrm{~m}$, where $\mathrm{D}$ is the diameter of the cylinder). Figure 2 shows that terms of the main diagonal in the matrix are similar to the coefficients of the isolated case, except for the small fluctuations, which appear due to the interaction in the array.

The symmetry of the layout configuration illustrated in Figure 1 makes the coefficients to be symmetric with respect to the y axis $(1=3 \& 4=5)$. Therefore, hydrodynamic coefficients for the devices 1 and 3, and 4 and 5 in the array are identical, as shown in Figure 2 .

Time average absorbed power by the array of $\mathrm{N}$ devices is calculated as follows in regular waves,

$$
P_{\text {array }}(\omega)=\frac{1}{2} \omega^{2} \operatorname{Re}\left(\hat{\delta}_{i} * \widetilde{B}_{P T O} \hat{\delta}_{i}\right)
$$

where, * expresses complex conjugate transpose between $\widetilde{B}_{P T O}$, a diagonal matrix, and $\hat{\delta}_{i}$, a $\mathrm{Nx} 1$ vector.

Power absorption of arrays subject to irregular waves is calculated in the same way as for single-devices following Equations (4) and (5), where the absorbed power $\left(P_{i}\right)$ in Equation (4) is given by Equation (7). All the hydrodynamic coefficients are obtained by means of the Ansys AQWA software, accounting for the mutual interactions within the array.

In this case, the PTO coefficient $\left(B_{P T O}\right)$ is the same for all the devices in the array, as 20] reported no significant improvement is obtained by individually optimising the PTO coefficient of each device. So $\widetilde{B}_{P T O}$ diagonal matrix can be replaced by the corresponding $B_{P T O}$ value for the isolated device and an identity matrix $(I)$ of the adequate order. The $B_{P T O}$ coefficient is optimized for each sea-state and cylinder type, as in the case with a single device.

\subsubsection{Gain factor (q)}

In the case of WEC arrays, the gain- or q-factor allows for the study of absorbed power variations in percentage terms, between that for an isolated 

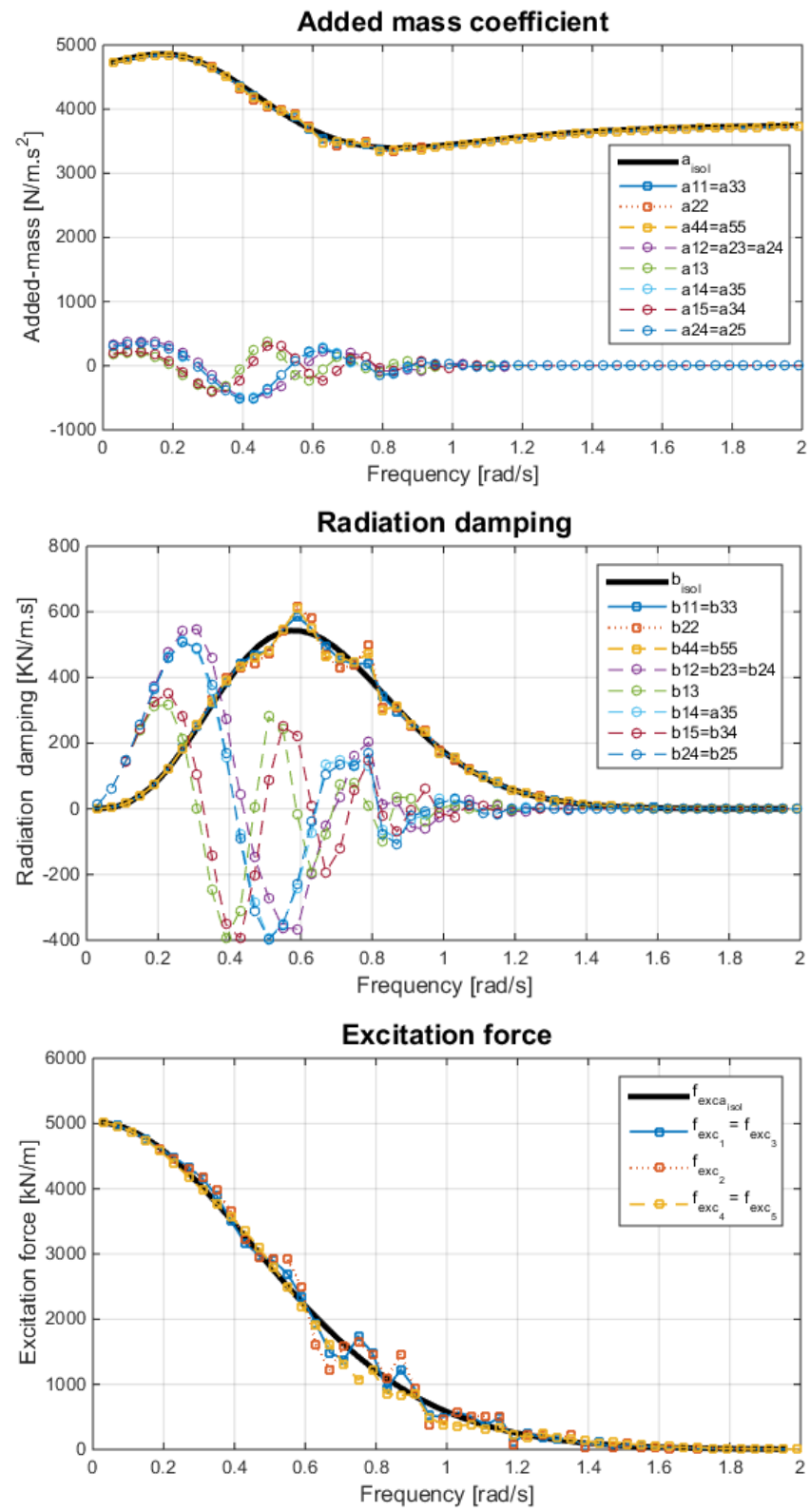

Figure 2: Hydrodynamic coefficients for a 5-device array comprises cylinders of ratio equal to 1 and inter-device spacing of 5 diameters, compared to the isolated case: added-mass (top), radiation damping (middle) and absolute value of the excitation force (bottom) 
device $\left(P_{\text {isolated }}\right)$ and those in an array $\left(P_{\text {array }}\right)$ as follows,

$$
q=\frac{P_{\text {array }}}{N P_{\text {isolated }}}
$$

where, $\mathrm{N}$ is the number of devices, $P_{\text {array }}$ is the total time-averaged absorbed power by the array and $P_{\text {isolated }}$ is the time-averaged absorbed power by the isolated device.

That way, the q-factor allows for comparison between the performance of

\section{Numerical examples}

\subsection{Device Geometry}

A vertical cylinder oscillating in heave has been chosen to represent the WEC geometry, in order to simplify the analysis, based on the dimensions of the new

ETO 6 device 33. However, in order to better analyse the behaviour of heaving cylinders in arrays, cylinders with different geometrical characteristics 
(I, II and III) have been studied in the simulations, using three slenderness (radius/draft) ratios: 0.5 (I), 1 (II) and 2 (III). To avoid scale effects and allow for a fair comparison, the masses of the three types of cylinders are kept the same, constraining the dimensions of the cylinders. Table 1 provides full details of the devices.

Table 1: Geometrical characteristics of the devices

\begin{tabular}{|c|c|c|c|c|}
\hline device & Radius $[\mathbf{m}]$ & Draft $[\mathbf{m}]$ & Mass $[\mathbf{K g}]$ & $T_{0}[\mathbf{s}]$ \\
\hline I & 10 & 20 & $6.45 \cdot 10^{6}$ & 10.2 \\
\hline II & 12.6 & 12.6 & $6.45 \cdot 10^{6}$ & 8.83 \\
\hline III & 15.9 & 7.9 & $6.45 \cdot 10^{6}$ & 7.93 \\
\hline
\end{tabular}

The behaviour of different cylinders is studied by analysing response amplitude operators (RAOs). Figure 3 shows the RAOs in heave of each type of cylinder with respect to wave frequency, where the maximum amplitude increases as the slenderness ratio decreases. The RAOs in Figure 3 include the effect of the power take-off. Hence, the more slender the cylinder is, the bigger its response to the waves is. However, an increase in response leads to narrower ranges of frequencies where the device responds. For all these reasons, the optimal ratio cannot be identified unequivocally at first blush.

The absorbed power of each type of cylinder is calculated by using annual mean power in irregular waves, as shown in Section 2 , and results are shown in Table 2 for the locations presented in Section 3.3 . The Bretschneider spectrum is used to represent conditions at all locations in order to simplify the study, as no more precise information is available about the spectral shape for each location.

Note that the cylinder III appears to the optimal one, regardless of the location and the wave characteristics, in agreement with results reported in 34. 


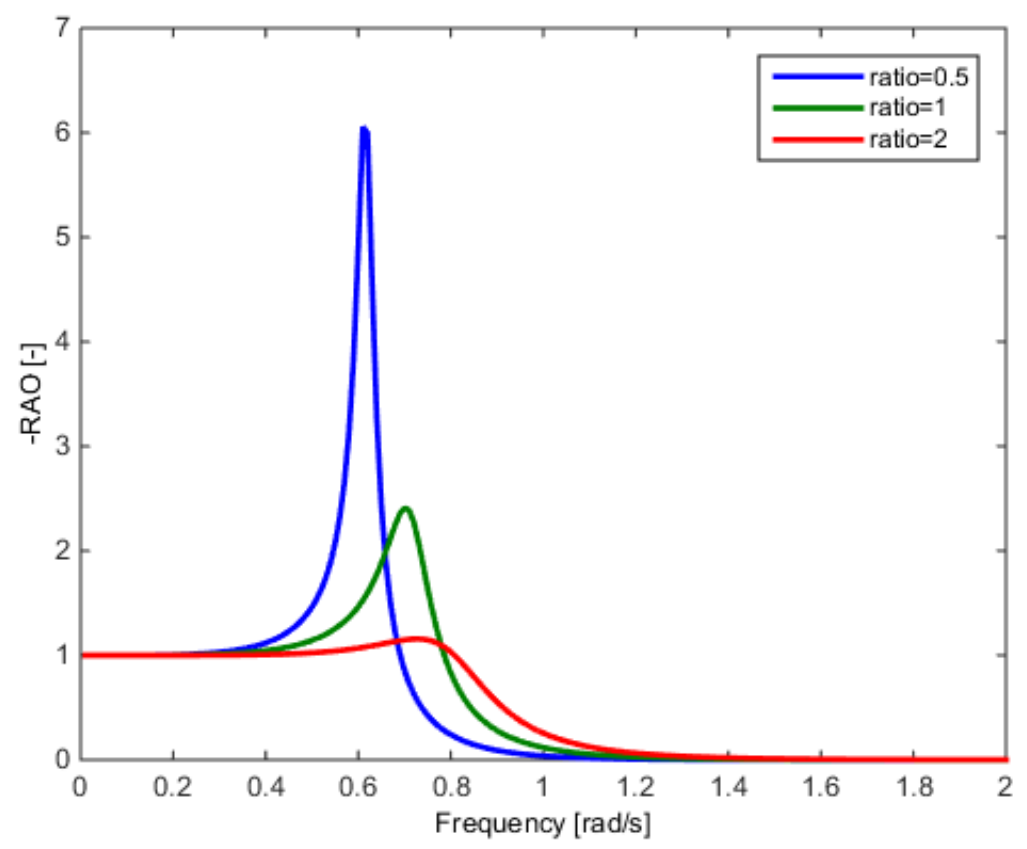

Figure 3: RAOs for the three different device geometries

Table 2: Absorbed power of isolated devices for each device type and location

\begin{tabular}{|c|c|c|c|c|}
\cline { 2 - 5 } \multicolumn{1}{c|}{} & \multicolumn{4}{c|}{ Annual mean absorbed power [kW] } \\
\hline device & Belmullet & Lisbon & BIMEP & SEMREV \\
\hline I & 220 & 133 & 75 & 57 \\
\hline II & 381 & 211 & 123 & 90 \\
\hline III & 543 & 305 & 175 & 131 \\
\hline
\end{tabular}

\subsection{Array layout and sizes}

In this paper, a single array layout is used: the staggered configuration. Devices of two consecutive rows are implemented in alternating positions, by horizontally displacing one of the two consecutive rows. That way, strong masking effects can be avoided as demonstrated numerically by [22] and experimentally by [13]. The horizontal (x) and vertical (y) inter-device spacings are identical

in the arrays used in this paper. An example of such an array with 5 devices is 
illustrated in Figure 1 .

Regarding the size of the arrays, it seems obvious that the more devices are in the array, the stronger the interactions are, as demonstrated in 35. However, this work only studies arrays up to 10 devices, which is considered by the authors of this paper to be a small array, since the future of wave energy devices appears to depend on farms with tens of devices, or perhaps hundreds, as mentioned by the some developers 36]. Therefore, it is interesting to analyse the effect of the hydrodynamic interactions in arrays of different number of devices, and consequently, different sizes.

In this paper, three array sizes are defined: small-, medium- and largearray. Generally, small arrays are considered those containing less than 10 devices, medium-size arrays consist of between 10 and 30 devices and large arrays include more than 30 devices, respecting the configuration illustrated in Figure 1 in all the cases. The goal of defining small, medium and large arrays 275 is to analyse the evolution of the interaction through the different sizes. The exact number of devices used in small-, medium- and large-size arrays is 5, 18 and 39 , respectively.

\subsection{Locations}

In order to cover a wider spectrum of the performance of wave energy devices, four different locations with different wave power resources are analysed in this study.

Table 3 shows the main characteristics of each location, while Figure 4 illustrates spectra occurrences as function of $H_{s}$ and $T_{p}$, and the geographical position of each location.

$T_{p}^{\prime}$ and $H_{s}^{\prime}$ are respectively the peak period and significant height with the highest frequency of occurrence at each location and $\lambda^{\prime}$ is the wavelength corresponding to the peak period with the highest frequency of occurrence. $J$ is the mean annual incoming wave energy per meter of wave front, obtained by multiplying the wave power resource $\left(P_{\text {wave }}\right)$ of each sea-state by its statisti- 

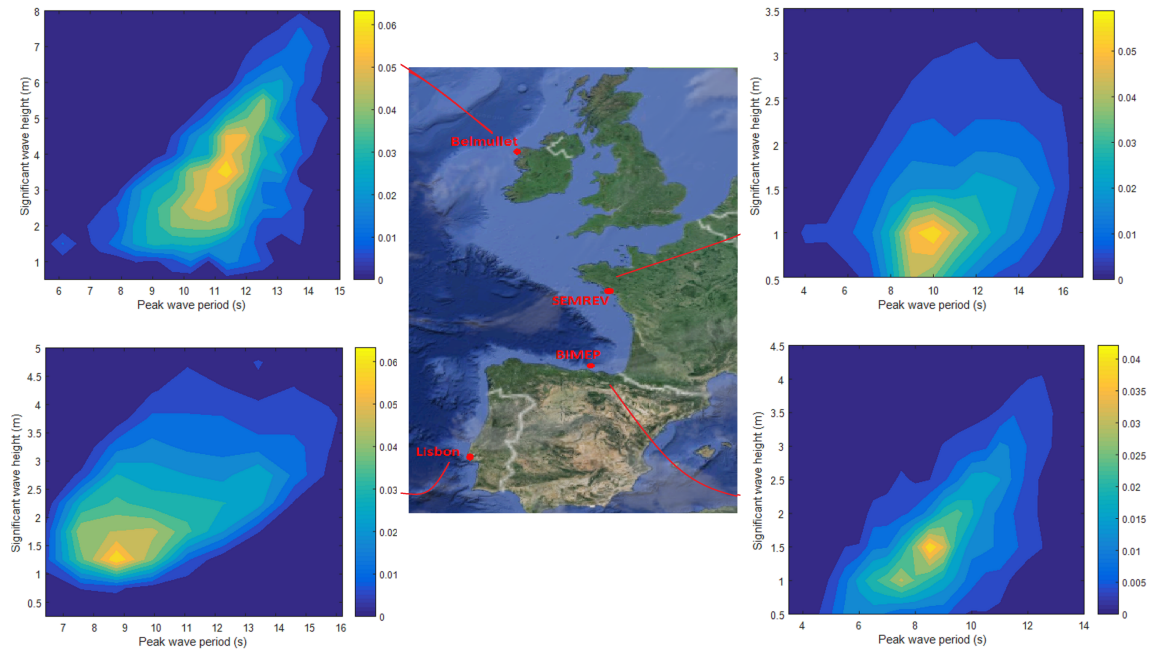

Figure 4: Different locations for the analysed arrays and their scattered diagrams

Table 3: Locations and corresponding spectral characteristics

\begin{tabular}{|c|c|c|c|c|}
\hline Location & $T_{p}^{\prime}[\mathbf{s}]$ & $H_{s}^{\prime}[\mathbf{m}]$ & $\lambda^{\prime}[\mathbf{m}]$ & $J[\mathrm{~kW} / \mathbf{m}]$ \\
\hline Belmullet & 11.3 & 3.5 & 200 & 78 \\
\hline Lisbon & 8.75 & 1.25 & 120 & 38 \\
\hline BIMEP & 8.5 & 1.5 & 110 & 22 \\
\hline SEMREV & 10 & 1 & 160 & 15 \\
\hline
\end{tabular}

follows,

$$
J=\sum_{\left(H_{s}, T_{p}\right)} P_{\text {wave }}\left(H_{s}, T_{p}\right) C\left(H_{s}, T_{p}\right)
$$

where the wave power resource of each sea-state is calculated from Equation (10) as shown in 30,

$$
P_{\text {wave }}=\frac{\rho g^{2} A(\omega)^{2}}{4 \omega}
$$

where $\rho$ is the water density and $g$ the acceleration due to gravity.

The selection of the test-sites is made with the aim of having as wide a representation of different wave power resources as possible. 


\section{Results}

Results are provided in terms of the previously described q-factor, since it is the parameter that best describes the hydrodynamic interaction in arrays.

values, a fair comparison between devices is guaranteed.

Table 4: Power absorption and hydrodynamic interaction characteristics of 5-device arrays

\begin{tabular}{|c|c|c|c|c|c|c|c|c|c|c|c|c|}
\multicolumn{1}{c|}{} & \multicolumn{3}{c|}{ Belmullet } & \multicolumn{3}{c|}{ Lisbon } & \multicolumn{3}{c|}{ BIMEP } & \multicolumn{3}{c|}{ SEMREV } \\
\hline device & $P_{\text {isol }}$ & $q_{M}$ & $q_{m}$ & $P_{\text {isol }}$ & $q_{M}$ & $q_{m}$ & $P_{\text {isol }}$ & $q_{M}$ & $q_{m}$ & $P_{i s o l}$ & $q_{M}$ & $q_{m}$ \\
\hline I & 1100 & 1.04 & 0.93 & 662 & 1.04 & 0.94 & 372 & 1.06 & 0.92 & 283 & 1.03 & 0.95 \\
\hline II & 1907 & 1.03 & 0.95 & 1056 & 1.03 & 0.95 & 614 & 1.03 & 0.93 & 541 & 1.02 & 0.96 \\
\hline III & 2715 & 1.05 & 0.95 & 1525 & 1.05 & 0.94 & 875 & 1.06 & 0.93 & 655 & 1.04 & 0.94 \\
\hline
\end{tabular}

Results are divided into three main groups, analysing the behaviour of each array size separately.

\subsection{Small farms}

Farms of up to 10 devices are considered as small farms. In this paper, small farms are represented by arrays of 5 devices implemented in two rows, three in the front and two in the back. Figure 1 illustrates the configuration of such a small array. 
Table 4 presents the main information to analyse hydrodynamic interactions the table are described as,

- $P_{\text {isol }}$ : Power of the array in $\mathrm{kW}$ if all the devices of the arrays were isolated devices;

- $q_{M}$ : The gain-factor value related to the inter-device distance in which power absorption is maximum; and

- $q_{m}$ : The gain-factor value related to the inter-device distance in which power absorption is minimum.

The behaviour of single-devices is repeated in small arrays, where power absorption increases with the slenderness ratio. The effect of the wave climate influence on the hydrodynamic interaction in an array. The hydrodynamic interaction is rather low in small farms, with a maximum impact of about $10 \%$ in all the cases, calculated by comparing the maximum and minimum values of the q-factor parameter in each case.

Analysing the hydrodynamic interaction in small farms as a function of the inter-device distance, the location where such interaction is maximum has been chosen: the BIMEP test site. Figure 5 shows q-factor values at each inter-device distance for the three devices defined in Section 3.1

It is important to note in Figure 5 that markers are exact q-factor values at each inter-device distance, while lines represent the trend of the q-factor through the different inter-device distances. These trend lines are obtained by using the smoothing spline method [37, where the $R$-square parameter of the fitting is always over 0.8. The horizontal black line $q=1$ represents the isolated case with neutral interactions or no interactions.

345 Hydrodynamic interactions with short inter-device distances are mostly destructive and highly inconsistent. Consequently, such short distances should be avoided. As the inter-device distance increases, hydrodynamic interactions 


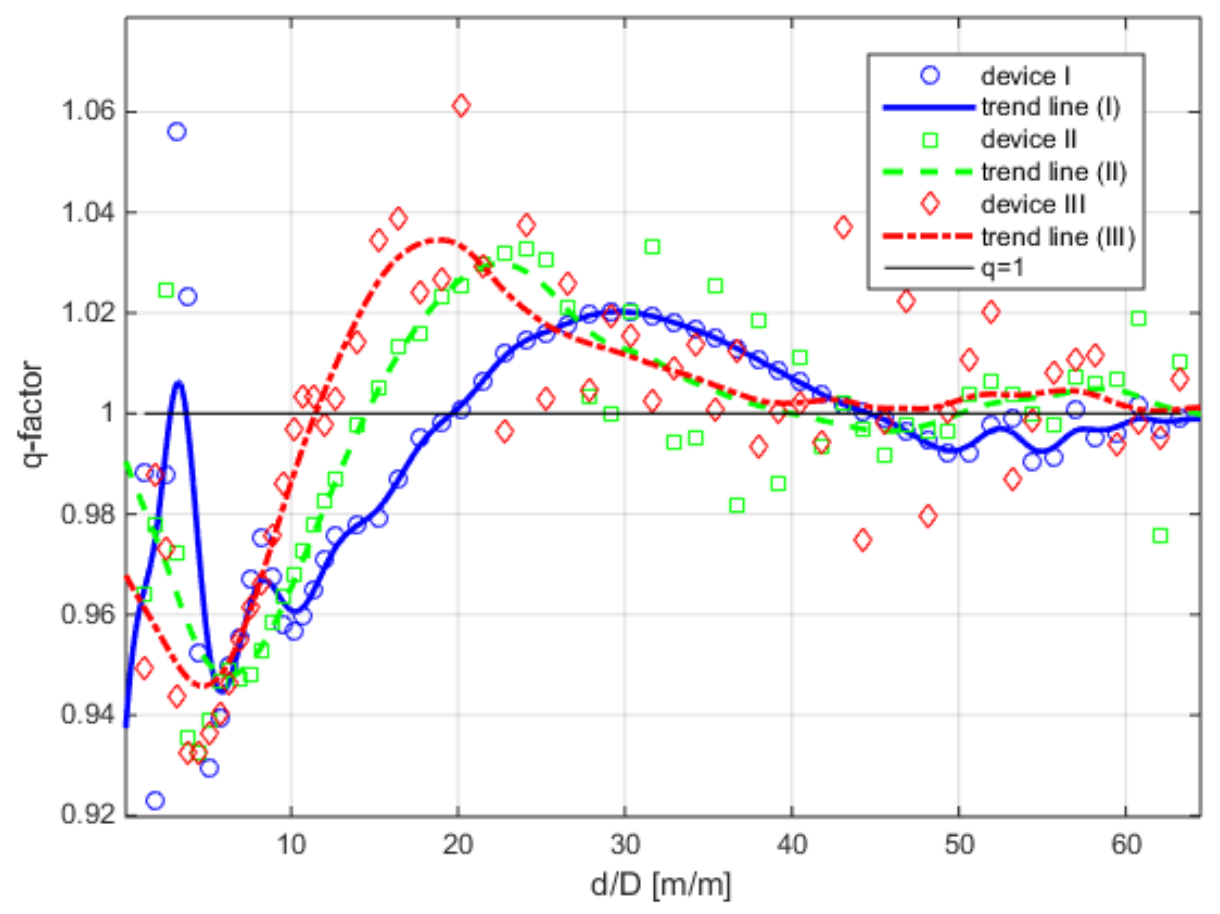

Figure 5: q-factor as function of normalised inter-device distance at BIMEP for small farms of different slenderness ratios.

become more consistent, especially for the device I, and constructive. Finally, with long inter-device distances, hydrodynamic interactions appear to vanish and devices in the farm behave as isolated devices.

Therefore, one can note three different regions with different interaction characteristics as a function of inter-device distances:

- Short inter-device distances: Mainly destructive and highly fluctuating hydrodynamic interactions.

- Intermediate inter-device distances: Constructive and relatively consistent hydrodynamic interactions.

- Long inter-device distances: Neutral and mostly consistent hydrodynamic interactions. 
Table 5: Power absorption and hydrodynamic interaction characteristics of 18-device arrays

\begin{tabular}{|c|c|c|c|c|c|c|c|c|c|c|c|c|}
\cline { 2 - 13 } \multicolumn{1}{c|}{} & \multicolumn{3}{c|}{ Belmullet } & \multicolumn{3}{c|}{ Lisbon } & \multicolumn{3}{c|}{ BIMEP } & \multicolumn{3}{c|}{ SEMREV } \\
\hline device & $P_{i s o l}$ & $q_{M}$ & $q_{m}$ & $P_{i s o l}$ & $q_{M}$ & $q_{m}$ & $P_{i s o l}$ & $q_{M}$ & $q_{m}$ & $P_{i s o l}$ & $q_{M}$ & $q_{m}$ \\
\hline I & 4523 & 1.05 & 0.85 & 2385 & 1.05 & 0.86 & 1340 & 1.08 & 0.84 & 1020 & 1.05 & 0.88 \\
\hline II & 6866 & 1.04 & 0.81 & 3801 & 1.05 & 0.80 & 2209 & 1.07 & 0.77 & 1625 & 1.04 & 0.82 \\
\hline III & 9778 & 1.06 & 0.73 & 5484 & 1.06 & 0.72 & 3146 & 1.09 & 0.71 & 2362 & 1.05 & 0.73 \\
\hline
\end{tabular}

Trend curves clearly illustrate the three regions and highlight the differences

360 sistency are characteristics that WEC developers want to exploit, inter-device distances of the intermediate-region seem to be the most adequate.

The lower the slenderness ratio, the more consistent the interaction is. However, the desirable inter-device region appears to start with longer inter-device interactions are in the intermediate region, but the stronger the fluctuations become.

Similar trends have been presented for small arrays [22, 26, 20, paying especial attention to the constructive peaks. However, the aforementioned three regions have never been identified so far in the literature. Very little attention was paid also to the effects of the array size or the slenderness of the devices in the array, as well as realistic wave climates.

\subsection{Medium farms}

Arrays of 18 devices, implemented in four rows following the staggered configuration illustrated in Figure 1, are used as medium-size farms. In order to compare the impact of hydrodynamic interactions in medium farms with the impact in small farms, the situations studied in small farms are again reproduced with 18 devices: devices of three slenderness ratios and wave climate data from four different locations.

380

Table 5 presents the main characteristics for the study of hydrodynamic interactions in medium farms. Regarding hydrodynamic interactions, differences 
between minimum and maximum q-factor values are considerably bigger for medium farms, with maximum values about $40 \%$. The influence of the location on the behaviour of the array appears again to be irrelevant.

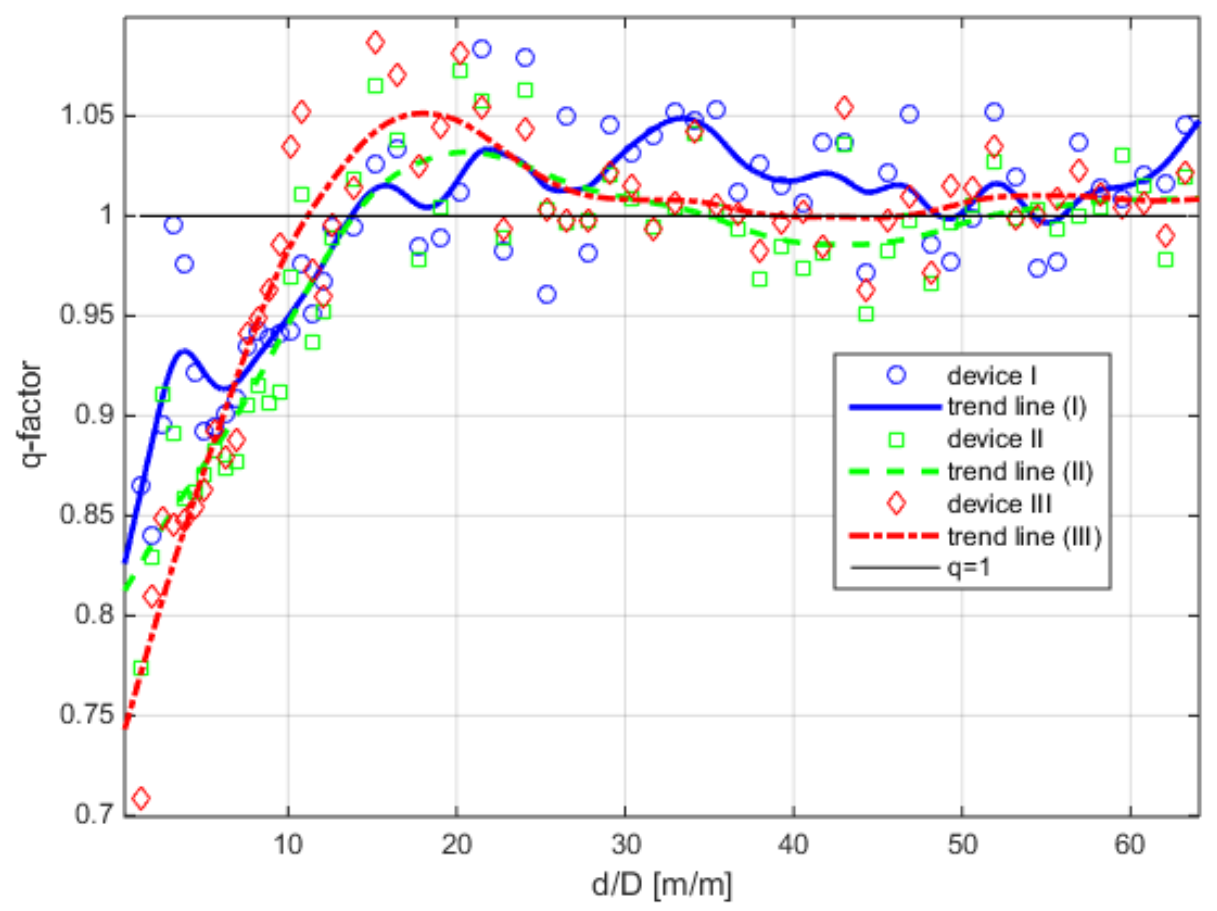

Figure 6: q-factor as function of normalised inter-device distance at BIMEP for medium farms of different slenderness ratios.

Figure 6 illustrates q-factor values for medium farms as a function of interdevice distance. In this case, fluctuations among consecutive simulations appear much stronger, rapidly moving from destructive to constructive interactions. However, trend curves still keep the desirable shape where the three regions mentioned previously in Section 4.1 are distinguishable. In contrast, the ideal characteristics of the intermediate region found in small farms, where interactions are constructive and mostly consistent, appear to weaken due to these strong fluctuations, especially in the case of the device I.

The short inter-device distances region in medium farms remains highly inconsistent and even more destructive than in small farms. The higher the 

inter-device distances. Nevertheless, similarly to the small farms, the intermediate region appears with shorter inter-device distances for devices with higher slenderness ratios.

\subsection{Large farms} with the size of the array. However, such impact does not increase linearly with the array size, as happens with small and medium farms, but may experience a saturation-like effect with large arrays.

Therefore, an array formed by 39 devices is studied. Since the computational been chosen. Although the highest ratio permits for higher power absorption, other aspects must also be considered when designing a WEC. WEC developers tend to prefer intermediate solutions $(r=1)$, as shown in [20] or [34], so the authors consider devices of type II for the study of large arrays.

Table 6: Power absorption characteristics of 39-device arrays using only the intermediate cylinder slenderness ratio $(r=1)$.

\begin{tabular}{|c|c|c|c|c|c|c|c|c|c|c|c|c|}
\cline { 2 - 13 } \multicolumn{1}{c|}{} & \multicolumn{3}{c|}{ Belmullet } & \multicolumn{3}{c|}{ Lisbon } & \multicolumn{3}{c|}{ BIMEP } & \multicolumn{3}{c|}{ SEMREV } \\
\hline device & $P_{i s o l}$ & $q_{M}$ & $q_{m}$ & $P_{i s o l}$ & $q_{M}$ & $q_{m}$ & $P_{i s o l}$ & $q_{M}$ & $q_{m}$ & $P_{i s o l}$ & $q_{M}$ & $q_{m}$ \\
\hline II & 14876 & 1.08 & 0.67 & 8235 & 1.07 & 0.67 & 4787 & 1.08 & 0.62 & 3521 & 1.06 & 0.68 \\
\hline
\end{tabular}

Table 6 illustrates that hydrodynamic interactions are stronger in large arrays, with maximum values of about 50\%, mainly due to stronger destructive interactions with very short inter-device distances. However, while the farm doubles the number of devices (increment of 100\%), the interaction only increases $10 \%$. Thus, a saturation-like effect has been demonstrated, as shown in Figure 7, where the impact of hydrodynamic interactions is plotted against array sizes with two increment trends for the impact of the interaction: a linear function and a quadratic function. The impact of hydrodynamic interaction in Figure 7 is referred to as the difference between the maximum and the minimum 
q-factor values in percentage for each array size.

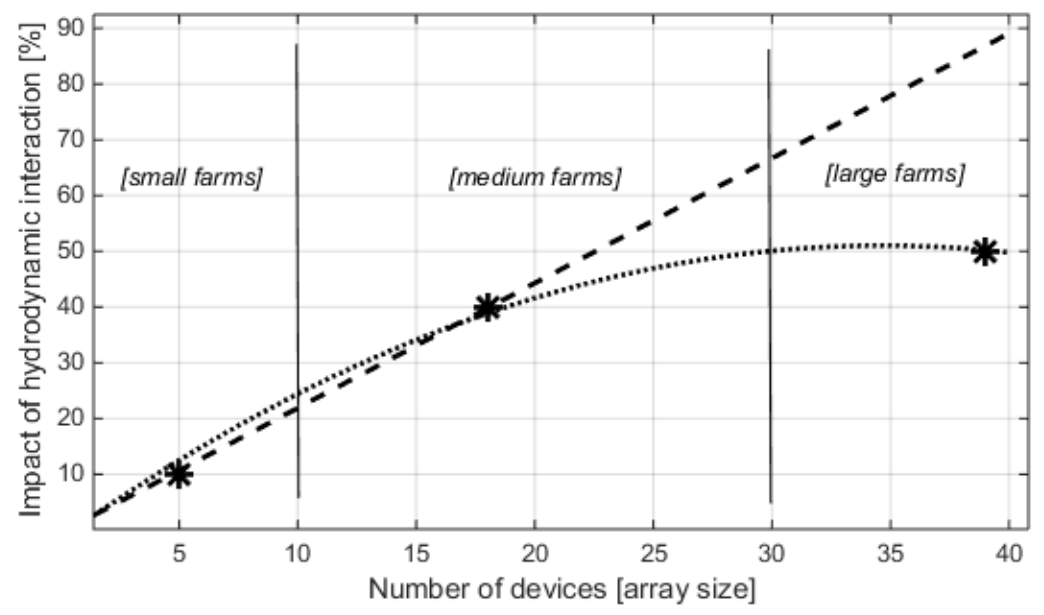

Figure 7: The impact of the hydrodynamic interaction for different array sizes.

This saturation-like effect means that after a number of devices in an array, the impact of hydrodynamic interaction may be constant, quantifiable as a constant percentage number.

With respect to the three regions identified especially in small farms, and to a lesser extent in medium farms, only the short inter-device region keeps its characteristics in large farms. The trend curve shown in Figure 8 clearly illustrates that the intermediate region completely disappears resulting in an inconsistent region where q-factor values strongly fluctuate around the neutral line $(q=1)$.

\section{Conclusion}

In this paper, the hydrodynamic impact of devices with different slenderness ratios and array sizes in wave energy farms is studied in four different realistic wave climates as a function of inter-device distance using the well-known qfactor.

The impact of wave climate in isolated devices is demonstrated to be important, while is practically irrelevant when studying the array configuration, since 


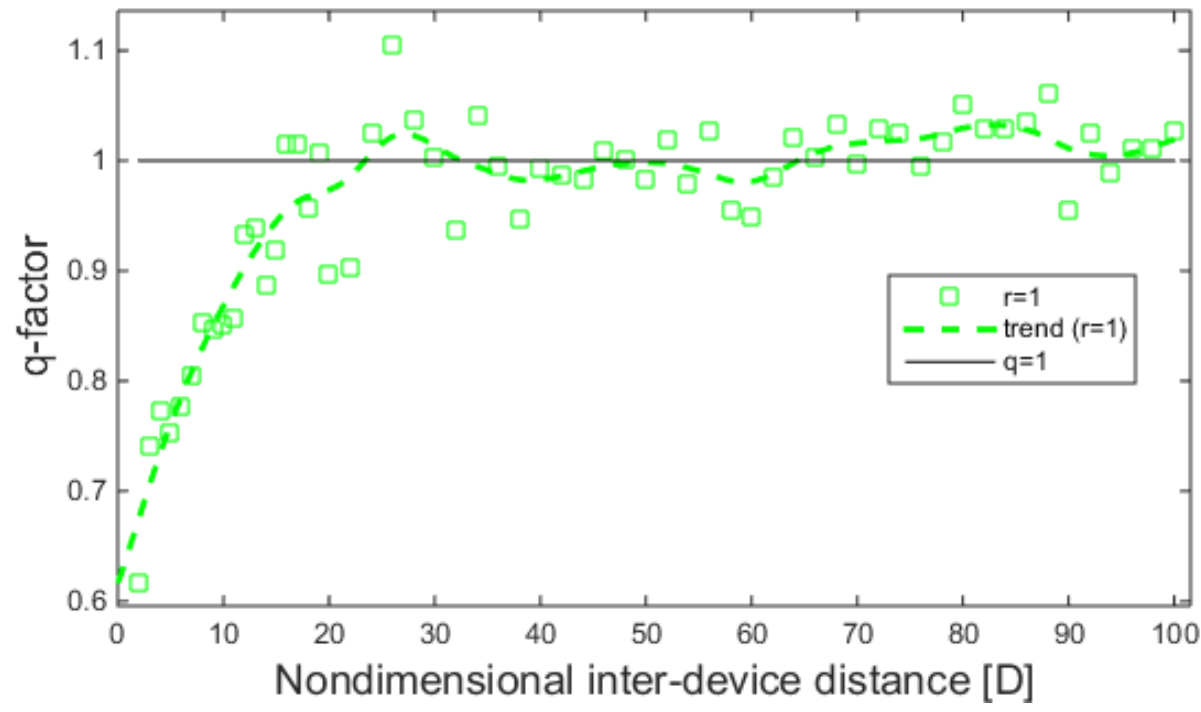

Figure 8: q-factor as function of normalised inter-device distance at BIMEP for a large farm of slenderness ratio $r=1$

variations in q-factor values and the shape of q-factor curves are insignificant. Therefore, the design of the isolated device should be optimised for a given location, but the array configuration can be designed independently. However, the most energetic periods of all the four wave climates used in this paper are similar, so a more in-depth analysis should be carried out before the irrelevance of wave climates can be confirmed.

Analysing farms of different sizes, it is found that hydrodynamic interactions do not increase linearly with the number of devices in the array forever. Instead, hydrodynamic interactions experience a saturation-like effect which limits their impact.

In small farms, three different regions with different interaction characteristics are identified: a highly destructive and inconsistent region for short distances, a mostly constructive and consistent region for intermediate distances, and a neutral consistent region for long distances. Characteristics of the intermediate region suggest that the optimal inter-device distance in a WEC array may be found in this region. However, characteristics of such regions disappear 
slowly as the size of the array increases, especially due to the intensification of fluctuations.

The slenderness of the devices implemented in the farm appears to be relarray.

\section{Acknowledgment}

This project was possible thanks to a collaboration between the University of ENSTA Bretagne in Brest (France) and the area of Marine Renewable Energy at 475 TECNALIA. Tecnalia's authors gratefully acknowledge the support of Basque Government ELKARTEK 2015 program -grant KK-2015/00097- that made this work possible. 


\section{References}

[1] G. Mork, S. Barstow, A. Kabuth, M. Pontes, Assesing the global wave energy potential, in: Procedings of 29th International Conference on Ocean, Offshore Mechanics and Artic Engineering (OMAE), China, 2010.

[2] K. Budar, J. Falnes, A resonant point absorber of ocean-wave power, Nature $256(1975) 478$.

[3] M. WAMIT Inc., WAMIT v7.0 manual (2013).

[5] NEMOH software (2014). URL https://lheea.ec-nantes.fr/doku.php/emo/nemoh/start

[6] K. Budal, Theory for absorption of wave power by a system of interacting bodies, Journal of ship research.

$490[7]$ J. Falnes, Radiation impedance matrix and optimum power absorption for interacting oscillators in surface waves, Applied Ocean Research 2 (2) (1980) $75-80$.

[8] P. McIver, Some hydrodynamic aspects of arrays of wave-energy devices, Applied Ocean Research 16 (2) (1994) 61-69.

[9] S. Mavrakos, P. McIver, Comparison of methods for computing hydrodynamic characteristics of arrays of wave power devices, Oceanographic Literature Review 9 (45) (1998) 1712.

[10] H. Kagemoto, D. K. Yue, Interactions among multiple three-dimensional bodies in water waves: an exact algebraic method, Journal of Fluid Mechanics 166 (1986) 189-209.

[11] A. Babarit, A impact of long separating distances on the energy production of two interacting wave energy converters, Ocean Engineering 37 (2010) 718-729. 
[12] M. Göteman, J. Engström, M. Eriksson, J. Isberg, M. Leijon, Methods of

[19] M. Folley, Section iii - wave energy converter array modelling techniques, in: M. Folley (Ed.), Numerical Modelling of Wave En-

a ergy Converters, Academic Press, 2016, pp. 151-225. doi:http: //dx.doi.org/10.1016/B978-0-12-803210-7.00010-4

530 URL http://www.sciencedirect.com/science/article/pii/ B9780128032107000104 
[20] P. Ricci, J.-B. Saulnier, A. Falcão, Point-absorber arrays: a configuration study off the portuguese west-coast, Proceedings of 7th European Wave and Tidal Energy Conference, Porto, Portugal, September (2007) 11-13.

[21] M. Folley, T. Whittaker, The effect of sub-optimal control and the spectral wave climate on the performance of wave energy converter arrays, Applied Ocean Research 31 (4) (2009) 260-266.

[22] B. Borgarino, A. Babarit, P. Ferrant, Impact of wave interactions effects on energy absorption in large arrays of wave energy converters, Ocean engineering 47 (2012) 79-88.

[23] J. Tissandier, A. Babarit, A. Clément, et al., Study of the smoothing effect on the power production in an array of searev wave energy converters., in: The Eighteenth International Offshore and Polar Engineering Conference, International Society of Offshore and Polar Engineers, 2008.

${ }_{545}$ [24] J. Singh, A. Babarit, A fast approach coupling boundary element method and plane wave approximation for wave interaction analysis in sparse arrays of wave energy converters, Ocean Engineering 85 (2014) 12-20.

[25] J. Engström, M. Eriksson, M. Göteman, J. Isberg, M. Leijon, Performance of large arrays of point absorbing direct-driven wave energy converters, Journal of Applied Physics 114 (20) (2013) 204502.

[26] A. De Andrés, R. Guanche, L. Meneses, C. Vidal, I. Losada, Factors that influence array layout on wave energy farms, Ocean Engineering 82 (2014) $32-41$.

[27] H. A. Wolgamot, C. J. Fitzgerald, Nonlinear hydrodynamic and real fluid effects on wave energy converters, Proceedings of the Institution of Mechanical Engineers, Part A: Journal of Power and Energy 229 (7) (2015) $772-794$.

[28] S. Chakrabarti, Handbook of Offshore Engineering, Vol. 1, Elsevier, 2005. 
[29] F. Arena, A. Ascanelli, V. Nava, D. Pavone, A. Romolo, Three-dimensional nonlinear random wave groups in intermediate water depth, Coastal engineering 55 (12) (2008) 1052-1061.

[30] J. Falnes, Ocean waves and oscillating systems: linear interactions including wave-energy extraction, Cambridge university press, 2002.

[31] F. Arena, V. Laface, G. Malara, A. Romolo, A. Viviano, V. Fiamma, G. Sannino, A. Carillo, Wave climate analysis for the design of wave energy harvesters in the mediterranean sea, Renewable Energy 77 (2015) 125 - 141. doi:http://dx.doi.org/10.1016/j.renene.2014.12.002. URL //www.sciencedirect.com/science/article/pii/ S0960148114008283

[32] G. Thomas, D. Evans, Arrays of three-dimensional wave-energy absorbers, Journal of Fluid Mechanics 108 (1981) 67-88.

[33] Carnegie wave energy, Available at http://www.carnegiewave.com/ (June 2016).

[34] I. Touzon, P. Ricci, M. Sanchez, G. Perez, F. Boscolo, Design, model and analysis of a combined semi-submersible floating wind turbine and wave energy point-absorber, in: In. proceedings of the 32nd International Conference on Ocean, Offshore and Arctic Engineering, Nantes, 2013.

[35] M. Vicente, A. Sarmento, Layout optimization of wave energy point absorber arrays, in: Proceedings of 10th European wave and tidal energy conference, Aalborg, 2013.

[36] Corpower ocean, Available at http://www.corpowerocean.com/ (June 2016).

[37] C. H. Reinsch, Smoothing by spline functions, Numerische mathematik 10 (3) (1967) 177-183. 